\title{
Erratum: "Magnitude and sign control of lithography-induced uniaxial anisotropy in ultrathin (Ga,Mn)As wires" \\ [Appl. Phys. Lett. 98, 083101 (2011)]
}

J. Shiogai, ${ }^{1,2, a)}$ D. Schuh, ${ }^{1}$ W. Wegscheider, ${ }^{1,3}$ M. Kohda, ${ }^{2,4}$ J. Nitta, ${ }^{2}$ and D. Weiss ${ }^{1}$

${ }_{1}^{1}$ Institute of Experimental and Applied Physics, University of Regensburg, 93053 Regensburg, Germany

${ }^{2}$ Department of Materials Science, Tohoku University, 980-8579 Sendai, Japan

${ }^{3}$ Department of Physics, ETH Zürich, 8093 Zürich, Switzerland

${ }^{4}$ PRESTO, Japan Science and Technology Agency, 332-0012 Kawaguchi, Japan

(Received 24 March 2011; accepted 1 April 2011; published online 18 April 2011)

[doi:10.1063/1.3581213]

In the originally published version of this letter the sentence on page 2 starting with "In the whole temperature range,..." is incomplete. Complete it should read as follows: "In the whole temperature range, the uniaxial AMR coefficient [the first term in Eq. (2)] for the $1-\mu \mathrm{m}$-wide wires is by a factor of 1.5 to 2 times larger than that for the $20-\mu \mathrm{m}$-wide wire [the first term in Eq. (1)].

${ }^{a)}$ Electronic mail: bltd5304@stu.material.tohoku.ac.jp. 